\title{
2020 vision: New insights on hypoxia imaging to assess cardiac and extra-cardiac active inflammatory sarcoidosis
}

\author{
Ronald G. Schwartz, MD, MS, FACC, FAHA, ABNM, MASNC, ${ }^{\mathrm{a}, \mathrm{b}}$ and \\ Himabindu Vidula, MD, MS, FACC ${ }^{\mathrm{a}}$ \\ a Division of Cardiology, Department of Medicine, University of Rochester Medical Center, \\ Rochester, NY \\ b Division of Nuclear Medicine, Department of Imaging Sciences, University of Rochester \\ Medical Center, Rochester, NY
}

Received Jan 4, 2020; accepted Jan 4, 2020

doi: $10.1007 / \mathrm{s} 12350-020-02032-4$

See related article, pp. 2141-2148

Myocardial perfusion and PET FDG metabolic imaging techniques provide state-of-the-art assessment of diagnosis, prognosis, and management of cardiac sarcoidosis (CS). Resting perfusion defects with PET or SPECT MPI reflect the extent and severity of fibrosis of non-caseating granulomas, while the leading edge of myocardial sarcoid inflammation is heralded by PET FDG uptake in the prolonged (12 to $18 \mathrm{~h}$ ) fasting state designed to suppress insulin-mediated physiologic myocardial glucose uptake which would otherwise overwhelm the weaker FDG signal of glycolysis of the inflammatory cell infiltration. ${ }^{1-3}$ Myocardial resting perfusion, ECG-gated function, and inflammation imaging data facilitate clinical decision making regarding optimal timing and intensity of immunosuppressive regimens and limit their formidable adverse effects while enhancing effective treatment to prevent or limit the development of major clinical manifestations of CS including heart failure, conduction defects, syncope, malignant ectopy, and sudden death.

The effective use of FDG imaging to evaluate active inflammatory CS poses several challenges for the centers of excellence conducting such studies and may

Reprint requests: Ronald G. Schwartz, MD, MS, FACC, FAHA, ABNM, MASNC, Division of Cardiology, Department of Medicine, University of Rochester Medical Center, 601 Elmwood Avenue, AC-G, Rochester, NY 14642-8679; ronald_schwartz@urmc.rochester.edu

J Nucl Cardiol 2021;28:2149-50.

$1071-3581 / \$ 34.00$

Copyright (c) 2020 American Society of Nuclear Cardiology. result in false-positive results. These include (1) the requirement for the prolonged fasting state of 18 hours for non-diabetic, or 12 hours for diabetic patients; (2) necessity for very low-carbohydrate meals prior to the prolonged fast, designed to deplete hepatic stores of glycogen and suppress physiologic pancreatic insulin release; (3) requirements for significant time and effort to provide communications and coordination by nuclear cardiology laboratory staff to patients scheduled for the studies; (4) unresolved controversies persist regarding the need for imposing activity restriction and use of heparin prior to FDG injection to minimize myocardial glucose extraction which could theoretically impair diagnostic accuracy and clinical effectiveness of the study; (5) physiologic diffuse myocardial FDG uptake associated with peritoneal dialysis ${ }^{4}$; and (6) regional or diffuse myocardial ischemia associated with epicardial $\mathrm{CAD}$ and/or microvascular disease that can trigger myocardial FDG uptake even after appropriate lowcarbohydrate dietary preparation and prolonged fasting, confounding the diagnosis of CS.

An alternative approach to detect active inflammatory $\mathrm{CS}$ is by imaging regional hypoxia with ${ }^{18} \mathrm{~F}$ fluoromisonidazole (FMISO) which is a hypoxia PET tracer that does not accumulate physiologically in the myocardium. ${ }^{5,6}$ In patients with malignancy, FMISO has been associated with Hypoxia Inducible Factor (HIF1alpha), a genetic marker related to glucose metabolism, transport, and VEGF-induced neovascularization that may be seen in inflammatory lesions as well. ${ }^{7}$ In the current issue of JNC, Furuya et al. report an early experience with this novel approach to detect hypoxia associated with active inflammatory sarcoidosis lesions detected by FDG PET. ${ }^{8}$ The investigators found most cardiac FDG lesions also showed FMISO uptake. A key 
advantage of FMISO hypoxia imaging of active CS inflammation is that no physiologic uptake of the tracer is noted in cardiac or extra-cardiac organs, and these patients did not require special dietary preparation. In fact, subjects in the study ate a typical lunch meal approximately one hour before PET CT was performed. PET imaging was performed 60 minutes after FDG administration and 4 hours after FMISO administration.

The accuracy of this approach appears promising, according to this initial report. Nine of 10 consecutive patients with sarcoidosis had cardiac involvement by traditional FDG PET performed 18 hrs after a low-carbohydrate meal. FMISO uptake was observed in 7 cardiac $(78 \%)$ and $6(55 \%)$ extra-cardiac lesions. The sensitivity and specificity of FMISO PET for diagnosing CS were $78 \%$ and $100 \%$, respectively. FMISO PET uptake was not observed in 2 patients with FDG uptake in the right ventricle or left atrium. SUVmax of FDG PET lesions was higher than that of FMISO PET. No lesion showed positive FMISO uptake but negative FDG uptake. The metabolic volume of lesions with FMISO uptake was larger than the lesions without the FMISO uptake.

Several limitations of the study merit consideration. The study sample of 10 patients, 9 of whom had CS is quite small. Notably, patients with coronary disease, diabetes, atrial fibrillation, bradycardia, tachycardia, cardiomyopathy, myocarditis, valvular heart disease, and cardiomegaly on chest X-ray were not included in the study. The diagnosis of CS was based on the diagnostic criteria established by the Japanese Society of Sarcoidosis and Other Granulomatous Disorders (JSSOG) ${ }^{9}$ rather than the Heart Rhythm Society ${ }^{10}$. Although 9 out of 10 patients had biopsy-proven extra-cardiac sarcoidosis, only 3 of these patients had biopsy proven CS. One patient received steroid therapy before imaging, and clinical outcome results in this small sample of subjects is not known.

The pioneering report of Furuya et al. stimulates new insights about the similarities of metabolic responses of hypoxic lesions of CS and malignancies that might be effectively imaged with FMISO PET or other metabolic tracers such as ${ }^{18}$ F-Fluorothymidine (FLT) or ${ }^{68}$ Ga-DOTANOC PET. ${ }^{11,12}$ Preliminary studies suggest these newer tracers correlate with fasting myocardial FDG PET lesions without the need for dietary and activity restriction, fasting, and heparin to suppress myocardial glucose uptake which may cause false-positive testing results. Will the hypoxia agents maintain or improve detection of CS and extra-cardiac sarcoidosis in patients with diabetes, renal failure patients on peritoneal dialysis, cardiomyopathy, atrial fibrillation, and other dysrhythmias? Whether tracers exploiting hypoxia or its downstream molecular responses can provide similar or greater diagnostic, prognostic and therapeutic response value to immuno- suppressive therapy for cardiac and extra-cardiac active inflammatory sarcoidosis compared to the gold standard FDG PET will require robust, well-powered clinical trials. As we begin 2020, the future of imaging molecular signatures of hypoxia in sarcoidosis appears bright.

\section{Disclosures}

Ronald G. Schwartz declares participation in the Astellas Speaking Bureau for non-branded presentations of appropriate use criteria and cardiac testing. Himabindu Vidula declares a research grant from Abbott for LVAD research.

\section{References}

1. Blankstein R. Cardiac positron emission tomography enhances prognostic assessments of patients with suspected cardiac sarcoidosis. J Am Coll Cardiol 2014;63:329-36.

2. Chareonthaitawee P, Beanlands RS, Chen W, Dorbala S, Miller EJ, Murthy VL, et al. Joint SNMMI-ASNC expert consensus document on the role of 18F-FDG PET/CT in cardiac sarcoid detection and therapy monitoring. J Nucl Cardiol 2017;24:174158. https://doi.org/10.1007/s12350-017-0978-9.

3. Schwartz RG, Malhotra S. Optimizing cardiac sarcoid imaging with FDG PET: Lessons from studies of physiologic regulation of myocardial fuel substrate utilization. J Nucl Cardiol 2018. https://d oi.org/10.1007/s12350-018-1438-x.

4. Gonzalez WO, Massera D, Travin MI. False-positive 13 N-ammonia/18 FDG PET for evaluation of cardiac sarcoidosis in a patient on peritoneal dialysis. J Nucl Cardiol 2015;22:1323-5. h ttps://doi.org/10.1007/s12350-015-0092-9.

5. Manabe O, Hirata K, Shozo O, et al. ${ }^{18}$ F-fluoromisonidazole (FMISO) PET may have the potential to detect cardiac sarcoidosis. J Nucl Cardiol 2017;24:329-31.

6. Sato J, Kitagawa Y, Yamazaki Y, et al. Advantage of FMISO-PET over FDG-PET for predicting histological response to preoperative chemotherapy in patients with oral squamous cell carcinoma. Eur J Nucl Med Mol Imaging 2014;41:2031-41.

7. Kizaka-Kondoh S, Tanaka S, Hiraoka M. Imaging and targeting of the hypoxia-inducible factor 1-active microenvironmen. J Toxicol Pathol 2009;22:93-100.

8. Furuya S, et al. ${ }^{18}$ F-FMISO PET/CT detects hypoxic lesions of cardiac and extra-cardiac involvement in patients with sarcoidosis. J Nucl Cardiol 2019. https://doi.org/10.1007/s12350-019-01976-6.

9. Ishida Y, Yoshinaga K, Miyagawa M, et al. Recommendations for ${ }^{18} \mathrm{~F}$-fluorodeoxyglucose positron emission tomography imaging for cardiac sarcoidosis: Japanese Society of Nuclear Cardiology recommendations. Ann Nucl Med 2014;28:393-403.

10. Birnie DH, Sauer WH, Bogun F, et al. HRS expert consensus statement on the diagnosis and management of arrhythmias associated with cardiac sarcoidosis. Heart Rhythm 2014;11:1304-23.

11. Martineau P, Pelletier-Galarneau M, Juneau D, et al. Imaging cardiac sarcoidosis with FLT PET compared with FDG perfusion PET. JACC Cardiovasc Imaging 2019; pii: S1936-878X(19)30612-6.

12. Gormsen LC, Haraldsen A, Kramer S, Dias AH, Kim WY, Borghammer P. A dual tracer ${ }^{68} \mathrm{Ga}$-DOTANOC PET/CT and ${ }^{18} \mathrm{~F}$ FDG PET/CT pilot study for detection of cardiac sarcoidosis. EJNMMI Res 2016;6:52.

Publisher's Note Springer Nature remains neutral with regard to jurisdictional claims in published maps and institutional affiliations. 